\title{
Mutational landscape and potential therapeutic targets for sporadic pancreatic neuroendocrine tumors based on target next-generation sequencing
}

\author{
KAILIAN ZHENG ${ }^{1 *}$, TAO LIU ${ }^{2 *}$, JIANGMAN ZHAO $^{3,4^{*}}$, PENG MENG $^{3,4}$, YUN BIAN $^{5}$, \\ CHENMING NI ${ }^{1}$, HUAN WANG ${ }^{1}$, YAQI PAN ${ }^{1}$, SHOUXIN WU ${ }^{3,4}$, HUI JIANG ${ }^{6}$ and GANG JIN ${ }^{1}$ \\ Departments of ${ }^{1}$ General Surgery and ${ }^{2}$ Emergency, Shanghai Changhai Hospital, Naval Medical University, \\ Shanghai 200433; ${ }^{3}$ Shanghai Zhangjiang Institute of Medical Innovation, Shanghai 201204; \\ ${ }^{4}$ Shanghai Biotecan Pharmaceuticals Co., Ltd.,Shanghai 201204; \\ Departments of ${ }^{5}$ Imaging and ${ }^{6}$ Pathology, Shanghai Changhai Hospital, \\ Naval Medical University, Shanghai 200433, P.R. China
}

Received May 19, 2020; Accepted September 11, 2020

DOI: $10.3892 /$ etm.2021.9859

\begin{abstract}
Pancreatic neuroendocrine tumor (PNET), a heterogenous type of neoplasm with limited treatment options, is relatively rare and to date, the genetic background has remained to be fully elucidated. The present study aimed to determine the mutational landscape of PNET with and without liver metastasis, as well as its clinical application value for treatment. Fresh tumor tissues were collected from 14 patients with PNET following surgery, 4 of whom had developed liver metastasis. Subsequently, targeted next-generation sequencing of 612 cancer-associated genes and comprehensive analysis were performed on the tumor tissues. The results identified 63 somatic mutations in 53 genes in the 14 patients with PNET, amongst which menin 1 was identified as the most recurrently mutated gene. The analysis also identified several novel recurrently mutated genes, including adrenoceptor alpha $2 \mathrm{~B}$, ARVCF delta catenin family member, carbamoyl-phosphate synthetase 2, aspartate transcarbamylase, and dihydroorotase and neuregulin 1. Among the 53 mutated genes, 11 were enriched in the PI3K/AKT signaling pathway (adjusted $\mathrm{P}=7.12 \times 10^{-5}$ ). In addition, 4 patients with $\mathrm{PNET}$ with
\end{abstract}

Correspondence to: Professor Gang Jin, Department of General Surgery, Shanghai Changhai Hospital, Naval Medical University, 168 Changhai Road, Shanghai 200433, P.R. China

E-mail: jingang@sohu.com

Dr Hui Jiang, Department of Pathology, Shanghai Changhai Hospital, Naval Medical University, 168 Changhai Road, Shanghai 200433, P.R. China

E-mail: jianghui5131@163.com

${ }^{*}$ Contributed equally

Key words: pancreatic neuroendocrine tumors, next-generation sequencing, liver metastasis, clinical actionable alterations liver metastasis had distinctly different mutational profiles compared with those without liver metastasis; 13 genes were discovered to be exclusively mutated in the liver metastasis group of the patients with PNET, including ATRX chromatin remodeler, thioredoxin reductase 2 , anus kinase $3, \mathrm{ARVCF}$ delta catenin family member, integrin subunit alpha $\mathrm{V}$ and RAD50 double strand break repair protein. In addition, two potentially actionable alterations in BRCA2 DNA repair-associated (p.Q548Q) and neurofibromin 1 (p.Q1188X) were identified using the OncoKB database. In conclusion, the present study generated a comprehensive mutational profile of 14 patients with PNET and further described the features of patients with liver metastasis, which highlights potential targets for drug development of PNET.

\section{Introduction}

Pancreatic neuroendocrine tumor (PNET) is a relatively rare type of neuroendocrine malignancy, originating from the islets of Langerhans in the pancreas. PNETs comprise $\sim 1-2 \%$ of all pancreatic neoplasms, demonstrating an annual incidence of 1 per 100,000 and a mortality rate of $60 \%$ (1-4). PNETs currently represent the second most common epithelial neoplasm after pancreatic ductal adenocarcinoma (PDAC) and are exhibiting a rising incidence and prevalence, particularly among Caucasians and Asians (4-6).

In total, $85 \%$ of PNETs are non-functional, presenting with a worse prognosis compared with hormone-secreting functional PNETs $(7,8)$. PNETs represent a diverse group of heterogeneous neoplasms with limited treatment options, which are morphologically and genetically different from PDACs and have far fewer mutations $(6,9)$. Although biological classifications may help define the various clinical behaviors and thus guide treatment, to the best of our knowledge, the genetic background of PNETs remains to be fully characterized.

Menin 1 (MEN1), death domain associated protein (DAXX), ATRX chromatin remodeler (ATRX) and mTOR 
signaling pathway genes, including phosphatase and tensin homolog (PTEN), TSC complex subunit 1, TSC complex subunit 2, DEP domain containing 5, GATOR1 subcomplex subunit and phosphatidylinositol-4,5-bisphosphate 3-kinase catalytic subunit alpha (PIK3CA), were previously identified to be commonly mutated in PNETs $(8,10)$. Additional novel germline mutations in the DNA repair genes, mutY DNA glycosylase, checkpoint kinase 2 and BRCA2 DNA repair-associated (BRCA2), and recurrent inactivating mutations or chromosomal rearrangements in chromatin-remodeling genes, including SET domain containing 2, histone lysine methyltransferase (SETD2), AT-rich interaction domain 1A (ARID1A) and lysine methyltransferase 2C (KMT2C), have also been reported (4). At the time of diagnosis, $\sim 60 \%$ of patients with nonfunctional PNETs have developed liver metastases, which results in a worse clinical outcome (11). Therefore, an improved understanding of the genetic background in PNET liver metastases may pave the way for the development of potential novel treatment strategies.

In the present study, 14 patients with PNETs were recruited and target next-generation sequencing (NGS) was performed on the primary fresh tumor tissues. The hybridization capture-based NGS panel, Biotecan PanCancer Panoramic Detection (12), which was designed according to the cancer-related database, clinical guidelines and high-quality references of solid tumors, covered the exons and promoter areas of the 612 cancer-associated genes $(2.75 \mathrm{M})$. Based on the sequencing data, a mutational profile of 14 patients with PNET was constructed and differences in the mutational profile between patients with and without liver metastasis were further investigated. The study also evaluated the utility of the NGS panel to guide the clinical management of patients with PNET.

\section{Materials and methods}

Patient samples. Fresh primary tumors were collected from 14 pathologically confirmed sporadic patients with PNETs following surgery at the Changhai Hospital (Shanghai, China) between February 2016 and September 2017. Images of H\&E-stained tumor tissues of two patients are exhibited in Fig. S1A and B. The percentage of Ki-67 nuclear staining (Ki-67 index; Fig. S1C-F) (13) was used to determine the grade of PNET (14). The histological diagnosis of tumors was performed and confirmed by two pathologists. None of the patients had received any therapeutic procedures, including chemotherapy or radiotherapy, prior to surgery. Samples were immediately frozen in liquid nitrogen and stored at $-80^{\circ} \mathrm{C}$ until further analysis. The clinicopathological features of the 14 patients are presented in Table I.

DNA extraction and quality control $(Q C)$. Genomic (g)DNA from the fresh frozen tumor tissues was extracted using a QIAamp DNA Mini kit (Qiagen $\mathrm{GmbH}$ ). The quantity and purity of the gDNA were assessed using a Qubit ${ }^{\circledR} 3.0$ fluorometer (Invitrogen; Thermo Fisher Scientific, Inc.) and a NanoDrop ND-1000 (Thermo Fisher Scientific, Inc.). The fragmentation status was evaluated using the Agilent 2200 TapeStation system using the Genomic DNA ScreenTape assay (Agilent Technologies, Inc.) to produce a DNA integrity number. An additional QC step to determine the fresh frozen tissue DNA integrity was performed using a multiplex PCR approach (15). In brief, $30 \mathrm{ng}$ gDNA was amplified using three different size sets of primers for the GAPDH gene (200-400 bp), and the concentration of the PCR products was determined using an Agilent 2100 Bioanalyzer instrument (Agilent Technologies, Inc.). Then, to estimate the fresh frozen tissue gDNA fragmentation, the average yield ratio value was determined by calculating the yield ratio of each amplicon compared with the Standard human genomic DNA (Promega Corproation; cat. no. G3041).

Library preparation, hybridization capture and amplification. A total of $300 \mathrm{ng}$ of each gDNA sample, which was based on Qubit quantification, was mechanically fragmented (duty factor 10\%; peak incident power $175 \mathrm{~W}$; cycles per burst 200; treatment time $240 \mathrm{sec}$; bath temperature $2-8^{\circ} \mathrm{C}$ ) on an E220 focused ultrasonicator (Covaris, Inc.). The target DNA fragment size is 150-200 bp. Subsequently, $200 \mathrm{ng}$ sheared gDNA was used to perform end repair, A-tailing and adapter ligation with a KAPA library preparation kit (Kapa Biosystems Inc.), according to the manufacturer's protocol. Subsequently, the libraries were captured using Agilent SureSelect XT custom 0.5-2.9M probes (Agilent Technologies, Inc.) and amplified.

Illumina sequencing. Following QC and quantification using the Agilent 2100 bioanalyzer (Agilent Technologies, Inc.) and a Qubit 2.0 fluorometer (Invitrogen; Thermo Fisher Scientific, Inc.), the libraries were sequenced on the Illumina Next 500 platform (Illumina, Inc.) in high-output mode, with $2 \times 75$ cycles.

Bioinformatics analysis. Clean data were obtained after filtering out the adapters and reads with a proportion of $\mathrm{N}>10 \%$, with $\mathrm{N}$ being the unidentified bases in the sequencing process, using fastp (fastp 0.19.4) (16). Low-quality bases (Phred score $<15$ ) were excised from the 3 ' ends of reads. Reads with length of $<36$ bp after excision were removed. The clean data were mapped to the reference human genome (University of California Santa Cruz ID: hg19; GenBank accession no. GCA_000001405.1) using the BWA alignment algorithm (BWA. 0.7.17) (17). The alignment in the SAM format was converted to BAM files using SAMtools (Samtools 1.9.0) (18). Next, the genome analysis toolkit (GATK; v4.0.2.1) (19) was used for sorting, duplicate marking and base recalibration. The final BAM files were analyzed using QualiMap v.2.2.1 (20) to provide an overall overview of the data, including mapped reads, mean mapping quality and mean coverage. The variants [single nucleotide variants (SNV) and insertion (Ins)-deletion (Del) mutations (InDels)] were called for unpaired tumor sequences with 40 pooled blood samples (from healthy individuals) using the GATK mutect2 tumor-only mode with the parameter af-alleles-not-in-resource $0.00025 \%$, and the germline mutations and contaminations were filtered out using GATK FilterMutectCalls with parameters (max-germline-posterior 0.995). Somatic variants were annotated using the ANNOVAR software tool.

The following filter conditions were used to identify the candidate somatic alterations: i) all variations with COSMIC 
Table I. Summary of the baseline data of the patients with pancreatic NET $(n=14)$.

\begin{tabular}{lc}
\hline Variables & Value \\
\hline Age (years) & $50(28-65)$ \\
Male, sex & $7(50)$ \\
Functioning hormone secretion status & $0(0)$ \\
Location & \\
Head & 4 \\
Tail & 10 \\
Local invasion & \\
Nerve invasion & 4 \\
Vascular tumor thrombus & 4 \\
Lymph node metastasis & \\
Yes & 7 \\
No & 7 \\
Liver metastasis & \\
Yes & 4 \\
No & 10 \\
WHO classification & \\
NET G1 & 1 \\
NET G2 & \\
NET G3 & 2 \\
\hline Vals & \\
\hline
\end{tabular}

Values are expressed as the median (range), $\mathrm{n}$ or n (\%). WHO, World Health Organization; NET, neuroendocrine tumor.

evidence (http://cancer.sanger.ac.uk/cosmic) were retained; ii) the variants with a mutational allele frequency (maf) $>0.001$ in the 1,000 Genomes databases (1,000 Genomes Project Consortium; https://www.internationalgenome. org/) (21) were removed; iii) the functional benign variant sites predicted by PolyPhen-2 (22), SIFT (23), MutationTaster (24) and Combined Annotation-Dependent Depletion (25) were removed; and iv) only the following variant classifications were retained: Missense_Mutation, Nonsense_Mutation, Splice_Site, Frame_Shift_Ins, Frame_Shift_Del, In_Frame_ Ins, In_Frame_Del.

Statistical analysis. The mutational landscape across the cohort was created using the maftools package in $\mathrm{R}$ software $(\mathrm{R}$ 3.5.1, R Core Team; https://www.R-Project.org). Kyoto Encyclopedia of Genes and Genomes (KEGG) signaling pathway enrichment analysis (https://www.kegg.jp/) was performed to investigate the biological importance of the altered genes in all samples using the clusterProfiler package (26) in R software (R 3.5.1). A cut-off value of the adjusted P-value (p.adjust) of $<0.05$ was used to identify significantly enriched terms.

\section{Results}

Demographic and baseline characteristics of the patients. The demographic and baseline characteristics of the study participants are summarized in Table I. A total of 14 patients with PNET were analyzed in the present study, including
7 males and 7 females, with a median age of 50 years (age range, 28-65 years). In the study cohort, all tumors of patients were non-functional PNETs, which do not make hormones, or make hormones that do not cause a set of symptoms. Among them, 4 patients had tumors located in the pancreas head and the other 10 patients had tumors located in the pancreas tail. Regarding the World Health Organization grade (27), 1 patient had a grade $(\mathrm{G}) 1$ classified NET, 11 patients had G2 classified NETs and 2 patients had G3 classified NETs.

In 13 patients, the median follow-up time was 20 months (range, 5-38 months; Table SI). One patient (case no. 11) was lost to follow-up. Among the 14 patients who were examined for lymph node status, 7 patients were identified to have lymph node metastasis, while nerve invasion and vascular tumor thrombus were present in 4 patients, and 4 patients demonstrated distant metastasis to the liver. Furthermore, 2 patients also presented with type II diabetes mellitus (T2DM). The majority of the patients remained alive during the follow-up, except for 1 patient (case no. 8), who died at 15 months following surgery. In addition, 1 patient (case no. 13) developed liver metastasis at 7 months following surgery. The percentage of Ki-67 of 14 patients varied between 1 and $40 \%$ (Table SI).

Recurrent mutated genes in PNET with and without liver metastasis. Fig. S2 presents a summary of the maf files (Table SII) in the 14 patients with PNET. In total, 63 variations in 53 genes were identified, involving 5 variant classifications. For the SNVs, C>T was the most frequent SNV class. The median number of variants identified in the 14 samples was 5 (range, 1-8).

Fig. 1 presents the mutational profile of 14 patients with PNET, including the top 20 recurrently mutated genes, according to the presence or absence of liver metastasis. The mutated gene with the highest frequency was MEN1 (4/14; $29 \%$ ), which was consistent with the previously reported mutation rate of MEN1 (23.1-56\%) in PNET $(5,6)$. The other recurrent altered genes were revealed to be adrenoceptor alpha 2B (ADRA2B; 3/14; 21\%), ARVCF delta catenin family member (ARVCF; 2/14; 14\%), carbamoyl-phosphate synthetase 2, aspartate transcarbamylase, and dihydroorotase (CAD; $2 / 14 ; 14 \%)$, neuregulin 1 (NRG1; 2/14; 14\%), tumor protein $\mathrm{p} 53$ (TP53; 2/14; 14\%) and thioredoxin reductase 2 (TXNRD2; 2/14; 14\%) (Fig. 1). ATRX was only identified to be mutated in one case $(1 / 14 ; 7 \%)$, which was lower than the reported rate of somatic mutations $(13-25 \%)(5,28)$. Fig. 2 illustrates the frequency of mutations and the resulting protein structure of MEN1 and ADRA2B, while that of other genes (mutation frequency $\geq 2 / 14$ ), including ARVCF, CAD, NRG1, TP53 and TXNRD2, are presented in Fig. S3.

KEGG signaling pathway enrichment analysis of all somatically mutated genes. To further investigate the biological functions of the mutated genes, KEGG signaling pathway enrichment analyses were performed. Table SIII presents all of the significantly enriched signaling pathways associated with the mutated genes. The top 15 significantly altered signaling pathways based on p.adjust are presented in Fig. 3A. In total, 11 mutated genes were identified to be enriched in the PI3K/AKT signaling pathway (p.adjust $=7.12 \times 10^{-5}$ ), including fibroblast growth factor receptor 3 (FGFR3), Janus 


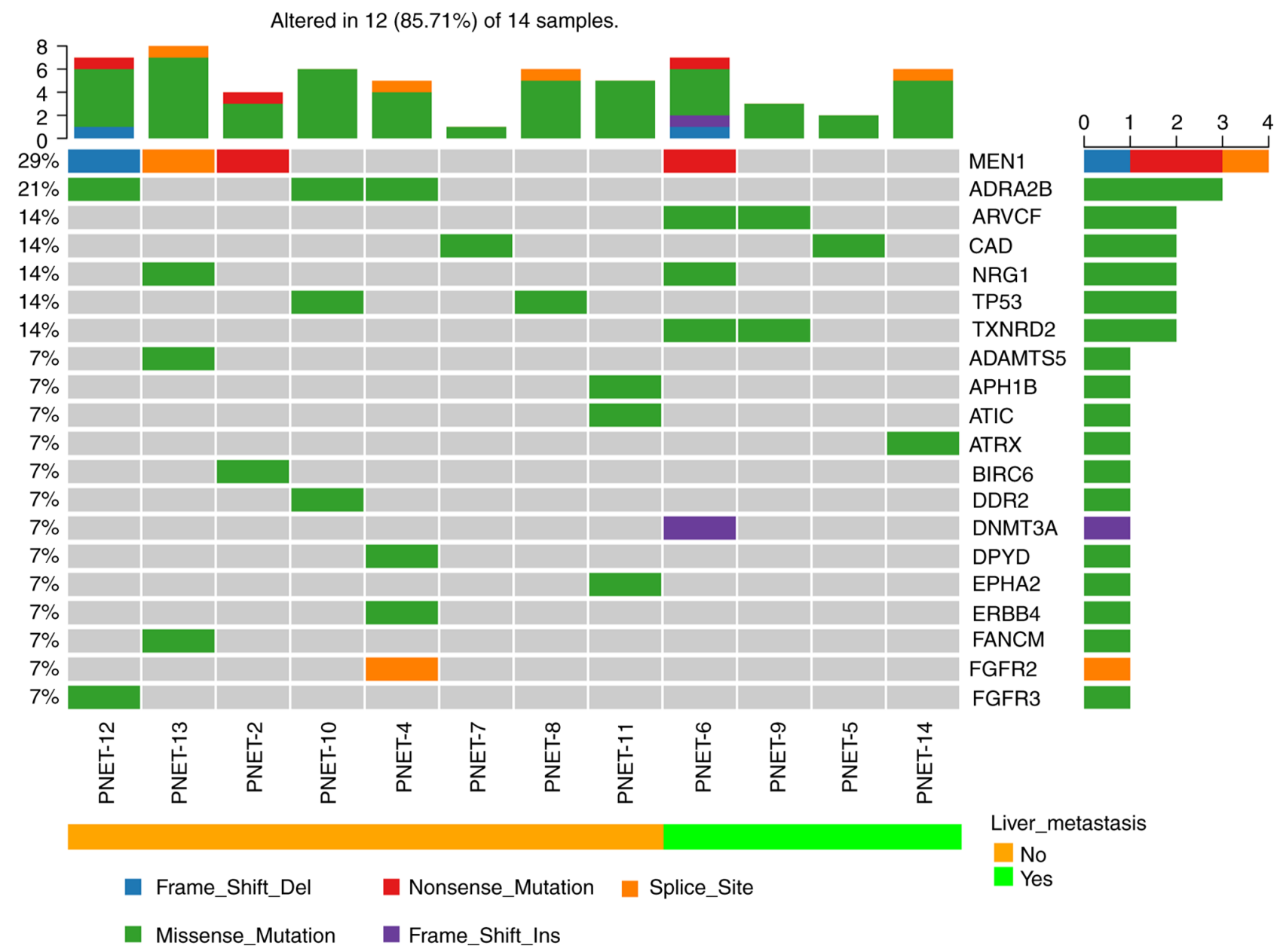

Figure 1. Mutational landscape and tumor mutational burden in 14 patients with PNET was determined using target next-generation sequencing. Patients were divided into liver metastasis and non-liver metastasis groups. PNET, pancreatic neuroendocrine tumor; MEN1, menin 1; ADRA2B, adrenoceptor alpha 2B; ARVCF, ARVCF delta catenin family member; CAD, carbamoyl-phosphate synthetase 2, aspartate transcarbamylase, and dihydroorotase; NRG1, neuregulin 1; TP53, tumor protein p53; TXNRD2, thioredoxin reductase 2; ADAMTS5, ADAM metallopeptidase with thrombospondin type 1 motif 5; APH1B, aph-1 homolog B, gamma-secretase subunit; ATIC,5-aminoimidazole-4-carboxamide ribonucleotide formyltransferase/IMPcyclohydrolase; ATRX,ATRXchromatin remodeler; BIRC6, baculoviral IAP repeat containing 6; DDR2, discoidin domain receptor tyrosine kinase 2; DNMT3A, DNA methyltransferase 3 alpha; DPYD, dihydropyrimidine dehydrogenase; EPHA2, EPH receptor A2; ERBB4, erb-b2 receptor tyrosine kinase 4; FANCM, FA complementation group M; FGFR2, fibroblast growth factor receptor 2; FGFR3, fibroblast growth factor receptor 3.

kinase 3 (JAK3), integrin subunit alpha V (ITGAV), EPH receptor A2 (EPHA2), phosphatidylinositol-4,5-bisphosphate 3-kinase catalytic subunit delta (PIK3CD), integrin subunit beta 1 (ITGB1), fibroblast growth factor receptor 2 (FGFR2), phosphoinositide-3-kinase regulatory subunit 3 (PIK3R3), erb-b2 receptor tyrosine kinase 4 (ERBB4), TP53 and PTEN. The PI3K/AKT signaling pathway is a well-established driver of carcinogenesis and has been reported in various types of cancer, such as gastric (29), breast (30) and colorectal cancer (31). Other well-known cancer-associated signaling pathways were also identified, including the focal adhesion, ErbB signaling pathway, Ras signaling pathway and MAPK signaling pathway. Endocrine-related signaling pathways, including the insulin resistance pathway, were also identified.

Differences in the mutational profile between two groups with/without liver metastasis. Three genes were discovered to be altered in the two groups, including MEN1, CAD and NRG1 (Fig. 3B). In addition, 13 genes were exclusively mutated in those patients with PNET who developed liver metastasis, including ATRX, TXNRD2, JAK3, ARVCF, ITGAV, RAD50 double strand break repair protein (RAD50), protein kinase $\mathrm{C}$ delta (PRKCD), tubulin alpha 3c (TUBA3C), DNA methyltransferase 3 alpha (DNMT3A), PIK3R3, integrin subunit alpha L (ITGAL), partner and localizer of BRCA2 (PALB2) and pyruvate dehydrogenase kinase 2 (PDK2). The results of the KEGG signaling pathway enrichment analysis of these 13 genes is presented in Table SIV, and it was discovered that the genes were involved in 'Homologous recombination', 'Chemokine signaling pathway' and 'Type II diabetes mellitus'.

Actionable genomic alterations in PNETs. To determine the clinical value of these genomic alterations, the OncoKBannotator (https://github.com/oncokb/oncokb-annotator) was used to group all alterations, without filtering the conditions, into various levels according to the evidence of clinical actionability. Altogether, two potentially actionable genomic alterations in BRCA2 (p.Q548Q) and neurofibromin 1 (NF1; p.Q1188X) were detected in patient nos. 14 and 8, respectively (Fig. 4). The evidence level for BRCA2 (p.Q548Q) for patients 


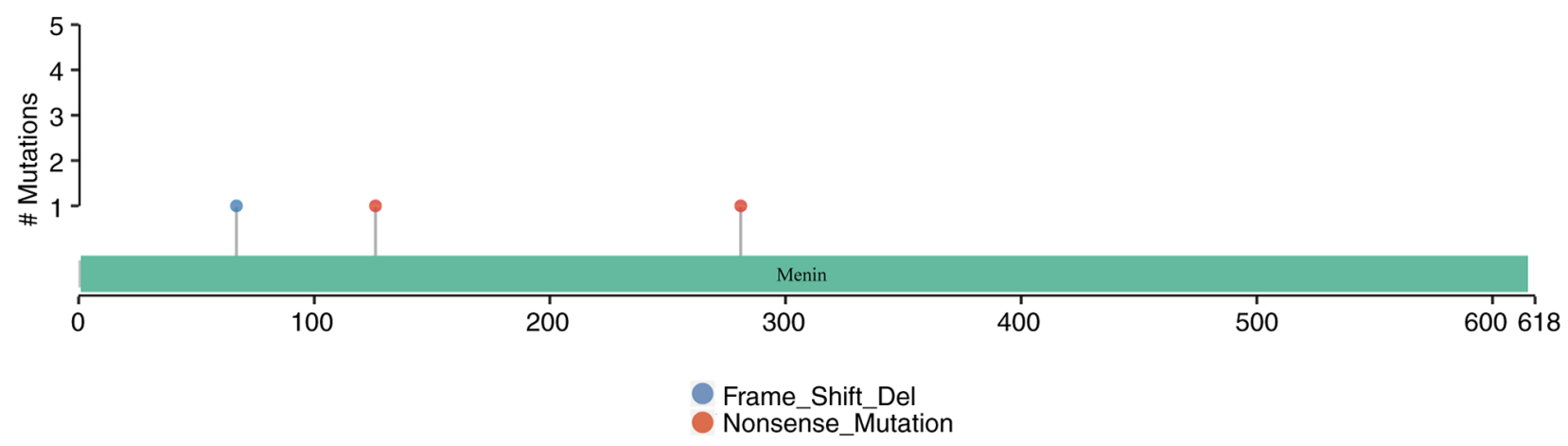

B

ADRA2B: [Somatic mutation rate:21.43\%] NM_000682

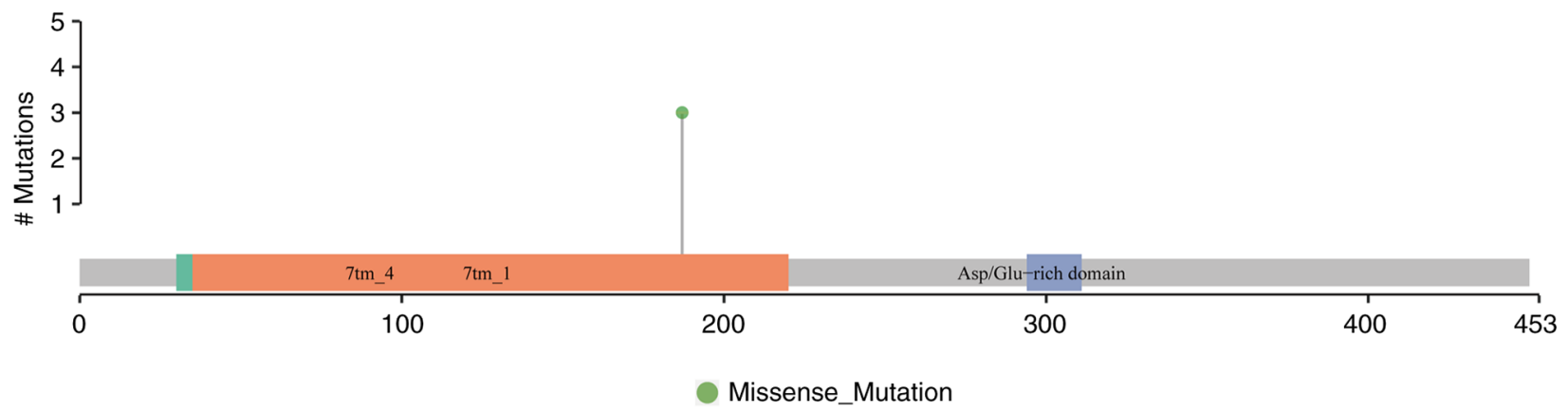

Figure 2. Proportion of mutations in (A) MEN1 and (B) ADRA2B. Del, deletion; MEN1, menin 1; ADRA2B, adrenoceptor alpha 2 B.

with PNET was LEVEL_2B, which was defined by OncoKB as a standard care biomarker recommended by the National Comprehensive Cancer Network (NCCN, https://education. nccn.org/) or other expert panels as predictive of a response to a Food and Drug Administration-approved drug; the corresponding drugs were niraparib, olaparib, rucaparib and talazoparib. The evidence level for NF1 (p.Q1188X) was LEVEL_4, which was defined as compelling biological evidence supporting the biomarker as being predictive of a response to a drug, and the corresponding drugs were cobimetinib and trametinib.

\section{Discussion}

The current treatments for PNET include medical therapy, surgery and radiotherapy, which are frequently unsuccessful, as the median survival time for patients with PNET is as low as $\sim 3.6$ years (2). Targeted therapies may provide a potential benefit for the patients; however, in order to develop these therapies, an improved understanding of the genetic landscape of PNET is required. In the present study, 14 PNET samples were analyzed in-depth by NGS-based gene panel sequencing; the study identified several novel recurrent genomic alterations in ADRA2B, ARVCF, CAD and NRG1, as well as the enrichment of altered genes in the PI3K/AKT signaling pathway.

Mutations in MEN1, DAXX/ATRX and mTOR signaling pathway genes have been frequently observed in $\sim 55-65 \%$ of patients with non-functional PNET (28). In the present study, a hybridization capture-based NGS panel was constructed to detect the genomic alterations in 612 cancer-associated genes. With this method, 14 cases of nonfunctional PNET with or without liver metastasis were sequenced. The highest frequency of the altered driver gene, MEN1, was also identified in the present study cohort, which was altered in four patients $(4 / 14 ; 29 \%)$. MEN1 encodes menin, a tumor suppressor associated with a syndrome known as multiple endocrine neoplasia type 1 (32). Its inactivation drives various phenotypes (4) which involves widespread transcriptional dysregulation via histone modifications (33), the activation of mTOR through AKT expression (34), the suppression of homologous recombination DNA damage response genes (35) and the dysregulation of telomerase reverse transcriptase (36). In addition, the altered genes were discovered to be highly enriched in the PI3K/AKT signaling pathway; this is consistent with other previous studies, which reported that mTOR pathway genes were frequently altered in PNETs and suggested the potential benefits of mTOR inhibitors, including everolimus, for Chinese patients with PNET.

In addition, the results of the present study revealed that the patients with PNETs that developed liver metastasis had distinct mutational profiles in the primary tumors compared with cases without liver metastasis. Lawrence et al (37) reported that there was a high consistency in the genome sequence, structure and expression between the primary tumors and hepatic metastases in one patient with PNET; 
A

B

C Regulation of actin cytoskeleton -

PI3K-Akt signaling pathway Rap1 signaling pathway Human T-cell leukemia virus 1 infection Small cell lung cancer Regulation of actin cytoskeleton Proteoglycans in cancer Cellular senescence . Signaling pathways regulating pluripotency of stem cells Neurotrophin signaling pathway EGFR tyrosine kinase inhibitor resistance -

Central carbon metabolism in cancer Prostate cancer Platinum drug resistance Endometrial cancer Endocrine resistance

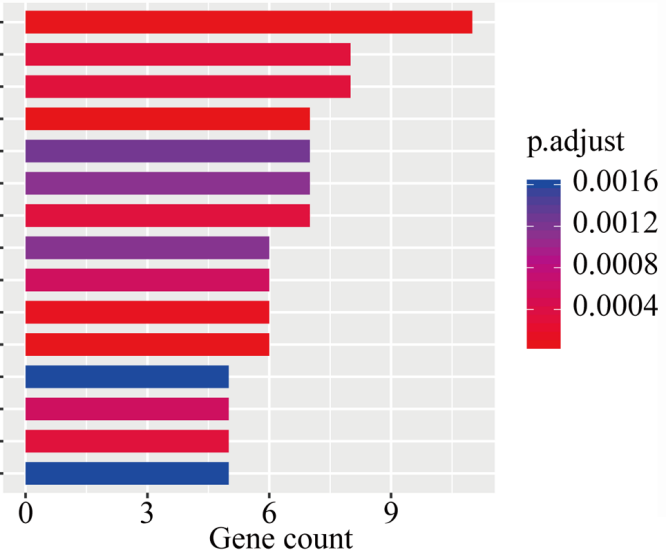

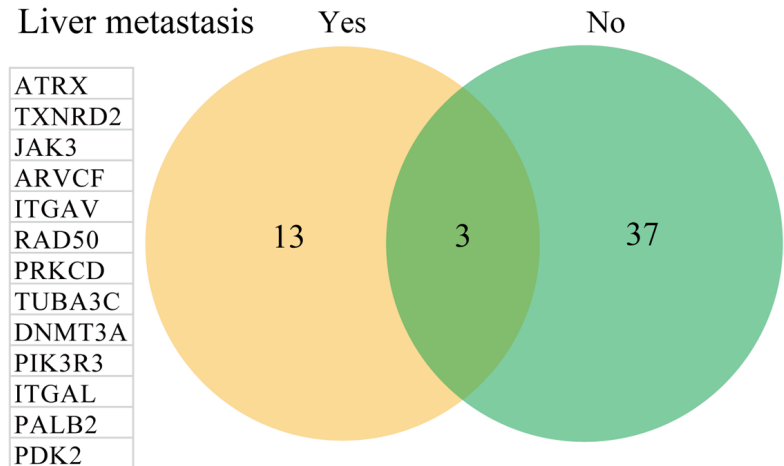

PDK2 Human T-cell leukemia virus 1 infection -

Epstein-Barr virus infection Chemokine signaling pathway Type II diabetes mellitus . Non-small cell lung cancer Homologous recombination -

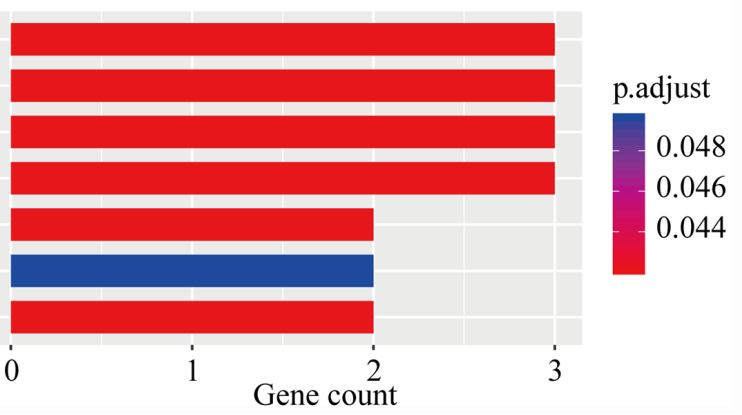

Figure 3. (A) Kyoto Encyclopedia of Genes and Genomes signaling pathway enrichment analysis of all somatically mutated genes. (B) Venn diagram of mutated genes between patients with PNET with or without liver metastasis. (C) Signaling pathways in which the 13 genes exclusively mutated in patients with PNET who had developed liver metastasis were enriched. PNET, pancreatic neuroendocrine tumor; p.adjust, adjusted P-value. ATRX, ATRX chromatin remodeler; TXNRD2, thioredoxin reductase 2; JAK3, Janus kinase 3; ARVCF, ARVCF delta catenin family member; ITGAV, integrin subunit alpha V; RAD50, RAD50 double strand break repair protein; PRKCD, protein kinase C delta; TUBA3C, tubulin alpha 3c; DNMT3A, DNA methyltransferase 3 alpha; PIK3R3, phosphoinositide-3-kinase regulatory subunit 3; ITGAL, integrin subunit alpha L; PALB2 partner and localizer of BRCA2; PDK2, pyruvate dehydrogenase kinase 2 .

however, the metastatic tumor lost certain variations or expression features observed in the primary tumor, whilst gaining certain de novo changes. In the present study, 13 genes were identified to be exclusively mutated in the patients who had developed liver metastasis, which were significantly enriched in 7 signaling pathways. The present study paid close attention to T2DM (p.adjust=0.030), which was enriched for 2 mutated genes, PRKCD and PIK3R3. Diabetes mellitus is an endocrine disease, which frequently occurs in patients with PNET. Long-standing diabetes mellitus has been discovered as a risk factor for PNET development $(38,39)$, and has been suggested to be associated with poor prognosis (40). Fan et al (41) determined that patients with PNET with T2DM were at an increased risk for tumor metastasis (odds ratio=2.81; $\mathrm{P}=0.001$ ) through investigating the clinicopathological characteristics of 299 patients with PNET. However, in the present study, the two patients with PNET and T2DM did not demonstrate liver metastasis. These results may reflect the substantial research in a large group of patients, which is further required to determine the relationship between T2DM and liver metastasis in PNETs.

In the present study, two potentially actionable genomic alterations in BRCA2 (p.Q548Q) and NF1 (p.Q1188X) were identified. BRCA2 is one of the most well-described cancer 


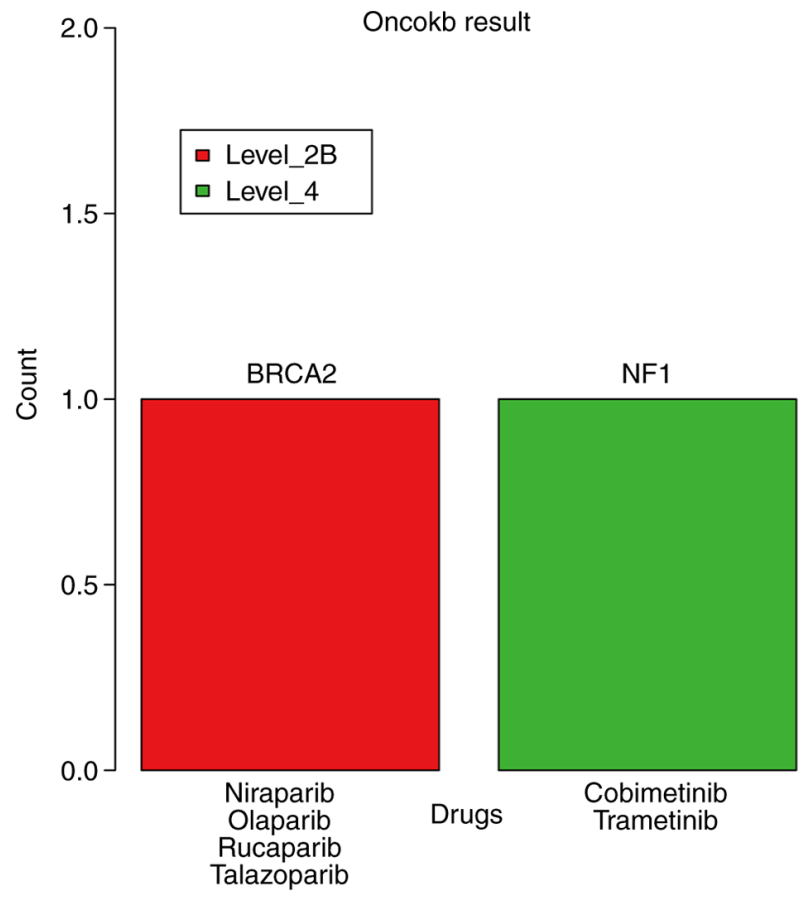

Figure 4. Clinical actionability of somatic alterations identified using target generation sequencing, including corresponding drugs and clinical evidence levels, according to the OncoKB database. NF1, neurofibromin 1; BRCA2, BRCA2 DNA repair-associated.

susceptibility genes, which serves critical roles in homologous DNA repair. High-risk germline or somatic mutations in BRCA2 usually lead to the defective repair of double-strand DNA breaks. BRCA2 mutations have been associated with multiple types of cancer, including breast, ovarian, pancreatic and prostate cancer (42). Several studies or case reports have reported the existence of BRCA2 germline mutations in PNET $(4,6,43)$, which is consistent with the results of the present study and highlights the germline contribution to clinically sporadic PNETs. NF1 resides on chromosome 17 and encodes neurofibromin, a $220 \mathrm{kDa}$ cytoplasmic protein, which is both a negative regulator of Ras and a positive regulator of adenylyl cyclase, the enzyme responsible for the generation of intracellular cyclic AMP (cAMP). Therefore, the loss of NF1 promotes the hyperactivation of downstream effector proteins of Ras signaling, including mTOR and MAPK kinase (MEK), and decreased intracellular cAMP levels. Germline mutations in NF1 were discovered to result in neurofibromatosis type 1, a complex autosomal-dominant disorder that affects multiple organ systems $(44,45)$. PNET is an uncommon clinical manifestation of the NF1 syndrome and is reported to be present in $<10 \%$ of cases (46). Previously, in an anecdotal report, the heterozygous germline mutation c.499 del TGTT was identified in a patient with NF1 syndrome and a well-differentiated pancreatic endocrine carcinoma (47). To the best of our knowledge, the presence of the NF1 mutant p.Q1188X has not been previously reported in nonfunctional PNET, which may be due to the difficulty to identify causative mutations in NF1 due to the large size of the gene ( 60 exons) and the diversity of clinical manifestations. However, whether this novel alteration of NF1 identified in the present study is targetable remains elusive. Further functional annotation is required to guide the clinical application of the MEK inhibitors, namely cobimetinib and trametinib.

Although the present study provided novel results, there are several limitations worthy of consideration. One limitation was the small sample size used in the study; only 14 patients with PNET were included and the present study was a retrospective study performed at a single center, which limits the generalizability of the results to the overall population of patients with PNET. Gene Ontology enrichment also should be used to investigate the biological functions of the mutated genes in the future large cohort study. Furthermore, functional studies in vitro were not performed to determine the biological effects of these mutated genes, which may also limit the significance of the results of the present study.

In conclusion, the present study identified several novel recurrent genomic alterations and confirmed the enrichment of gene alterations in the PI3K/AKT signaling pathway in a small cohort of Chinese patients with PNET. These results may shed light on opportunities for the personalized treatment of sporadic PNETs. Of note, patients with PNET who developed liver metastasis revealed distinct mutational profiles compared with cases without metastasis. Furthermore, two potentially clinically actionable genomic alterations were identified in BRCA2 and NF1, suggesting further clinical options of treatment targets in the future.

\section{Acknowledgements}

Not applicable.

\section{Funding}

The present study was supported by the Special Fund for Transformation and Application of Precision Medicine of the Second Military Medical University (grant no. 2017JZ41) and the Major Projects of Special Development Funds in Zhangjiang National Independent Innovation Demonstration Zone, Shanghai (grant no. ZJ2017-ZD-012).

\section{Availability of data and materials}

The datasets used and/or analyzed during the current study are available from the BioProject database (BioProject ID: PRJNA657158; http://www.ncbi.nlm.nih.gov/bioproject/657158).

\section{Authors' contributions}

KZ, TL, JZ, SW, HJ and GJ conceived and designed the study. YB, CN, HW and YP recruited the patients, performed the clinical examination, collected and organized the data. $\mathrm{KZ}, \mathrm{JZ}, \mathrm{PM}$ and SW analyzed and interpreted the data. KZ, TL and JZ wrote the manuscript. SW, HJ and GJ reviewed and edited the manuscript. All authors read and approved the manuscript.

\section{Ethics approval and consent to participate}

Ethical approval for the recruitment of human subjects was obtained from the Ethics Committee of Shanghai Changhai 
Hospital (Shanghai, China) and the protocol was in accordance with the ethical guidelines provided by the Declaration of Helsinki (1975). Written informed consent was obtained from each patient.

\section{Patient consent for publication}

Not applicable.

\section{Competing interests}

The authors declare that they have no competing interests.

\section{References}

1. Hopper AD, Jalal M and Munir A: Recent advances in the diagnosis and management of pancreatic neuroendocrine tumours. Frontline Gastroenterol 10: 269-274, 2019.

2. Dasari A, Shen C, Halperin D, Zhao B, Zhou S, Xu Y, Shih T and Yao JC: Trends in the incidence, prevalence, and survival outcomes in patients with neuroendocrine tumors in the United States. JAMA Oncol 3: 1335-1342, 2017.

3. Dumlu EG, Karakoc D and Özdemir A: Nonfunctional pancreatic neuroendocrine tumors: Advances in diagnosis, management, and controversies. Int Surg 100: 1089-1097, 2015.

4. Scarpa A, Chang DK, Nones K, Corbo V, Patch AM, Bailey P, Lawlor RT, Johns AL, Miller DK, Mafficini A, et al: Whole-genome landscape of pancreatic neuroendocrine tumours. Nature 543: 65-71, 2017.

5. Raj N, Shah R, Stadler Z, Mukherjee S, Chou J, Untch B, Li J, Kelly V, Saltz LB, Mandelker D, et al: Real-time genomic characterization of metastatic pancreatic neuroendocrine tumors has prognostic implications and identifies potential germline actionability. JCO Precis Oncol 2018: PO.17.00267, 2018.

6. Ji S, Yang W, Liu J, Zhao J, Chen L, Ni Q, Long J and Yu X: High throughput gene sequencing reveals altered landscape in DNA damage responses and chromatin remodeling in sporadic pancreatic neuroendocrine tumors. Pancreatology 18: 318-327, 2018.

7. Zhang J, Francois R, Iyer R, Seshadri M, Zajac-Kaye M and Hochwald SN: Current understanding of the molecular biology of pancreatic neuroendocrine tumors. J Natl Cancer Inst 105: $1005-1017,2013$

8. Batukbhai BDO and De Jesus-Acosta A: The molecular and clinical landscape of pancreatic neuroendocrine tumors. Pancreas 48: 9-21, 2019.

9. Chan CS, Laddha SV, Lewis PW, Koletsky MS, Robzyk K, Da Silva E, Torres PJ, Untch BR, Li J, Bose P, et al: ATRX, DAXX or MEN1 mutant pancreatic neuroendocrine tumors are a distinct alpha-cell signature subgroup. Nat Commun 9: 4158, 2018.

10. Pipinikas CP, Berner AM, Sposito T and Thirlwell C: The evolving (epi)genetic landscape of pancreatic neuroendocrine tumours. Endocr Relat Cancer 26: R519-R544, 2019.

11. < gutjnl-2018-insulinomas and non-functional PNETs.pdf>.

12. Cai H, Jing C, Chang X, Ding D, Han T, Yang J, Lu Z, Hu X, Liu Z, Wang J, et al: Mutational landscape of gastric cancer and clinical application of genomic profiling based on target next-generation sequencing. J Transl Med 17: 189, 2019.

13. Rindi G, Bordi C, La Rosa S, Solcia E and Fave GD; Gruppo Italiano Patologi Apparato Digerente (GIPAD); Società Italiana di Anatomia Patologica e Citopatologia Diagnostica/International Academy of Pathology, Italian division (SIAPEC/IAP): Gastroenteropancreatic (neuro)endocrine neoplasms: The histology report. Dig Liver Dis 43 (Suppl 4): S356-S360, 2011.

14. Halfdanarson TR, Strosberg JR, Tang L, Bellizzi AM, Bergsland EK, O'Dorisio TM, Halperin DM, Fishbein L, Eads J, Hope TA, et al: The North American neuroendocrine tumor society consensus guidelines for surveillance and medical management of pancreatic neuroendocrine tumors. Pancreas 49: 863-881, 2020.

15. Clavé S, Gimeno J, Muñoz-Mármol AM, Vidal J, Reguart N, Carcereny E, Pijuan L, Menéndez S, Taus Á, Mate JL, et al: ROS1 copy number alterations are frequent in non-small cell lung cancer. Oncotarget 7: 8019-8028, 2016.
16. Chen S, Zhou Y, Chen Y and Gu J: fastp: An ultra-fast all-in-one FASTQ preprocessor. Bioinformatics 34: i884-i890, 2018.

17. Li H and Durbin R: Fast and accurate long-read alignment with burrows-wheeler transform. Bioinformatics 26: 589-595, 2010.

18. Li H, Handsaker B, Wysoker A, Fennell T, Ruan J, Homer N, Marth G, Abecasis G and Durbin R; 1000 Genome Project Data Processing Subgroup: The sequence alignment/map format and SAMtools. Bioinformatics 25: 2078-2079, 2009.

19. McKenna A, Hanna M, Banks E, Sivachenko A, Cibulskis K, Kernytsky A, Garimella K, Altshuler D, Gabriel S, Daly M and DePristo MA: The genome analysis toolkit: A MapReduce framework for analyzing next-generation DNA sequencing data. Genome Res 20: 1297-1303, 2010.

20. García-Alcalde F, Okonechnikov K, Carbonell J, Cruz LM, Götz S, Tarazona S, Dopazo J, Meyer TF and Conesa A: Qualimap: Evaluating next-generation sequencing alignment data. Bioinformatics 28: 2678-2679, 2012.

21. 1000 Genomes Project Consortium, Auton A, Brooks LD, Durbin RM, Garrison EP, Kang HM, Korbel JO, Marchini JL, McCarthy S, McVean GA and Abecasis GR: A global reference for human genetic variation. Nature 526: 68-74, 2015.

22. Adzhubei I, Jordan DM and Sunyaev SR: Predicting functional effect of human missense mutations using PolyPhen-2. Curr Protoc Hum Genet Chapter 7: Unit7.20, 2013.

23. Ng PC and Henikoff S: SIFT: Predicting amino acid changes that affect protein function. Nucleic Acids Res 31: 3812-3814, 2003.

24. Schwarz JM, Rödelsperger C, Schuelke M and Seelow D: MutationTaster evaluates disease-causing potential of sequence alterations. Nat Methods 7: 575-576, 2010.

25. Kircher M, Witten DM, Jain P, O'Roak BJ, Cooper GM and Shendure J: A general framework for estimating the relative pathogenicity of human genetic variants. Nat Genet 46: 310-315, 2014.

26. Yu G, Wang LG, Han Y and He QY: clusterProfiler: An R package for comparing biological themes among gene clusters. OMICS 16: 284-287, 2012.

27. Nagtegaal ID, Odze RD, Klimstra D, Paradis V, Rugge M, Schirmacher P, Washington KM, Carneiro F and Cree IA; WHO Classification of Tumours Editorial Board: The 2019 WHO classification of tumours of the digestive system. Histopathology 76: 182-188, 2020.

28. Hong X, Qiao S, Li F, Wang W, Jiang R, Wu H, Chen H, Liu L, Peng J, Wang J, et al: Whole-genome sequencing reveals distinct genetic bases for insulinomas and non-functional pancreatic neuroendocrine tumours: Leading to a new classification system. Gut 69: 877-887, 2020.

29. Cancer Genome Atlas Research Network: Comprehensive molecular characterization of gastric adenocarcinoma. Nature 513: 202-209, 2014.

30. Cancer Genome Atlas Network: Comprehensive molecular portraits of human breast tumours. Nature 490: 61-70, 2012.

31. Cancer Genome Atlas Network: Comprehensive molecular characterization of human colon and rectal cancer. Nature 487: 330-337, 2012.

32. Beijers HJBH, Stikkelbroeck NML, Mensenkamp AR, Pfundt R, van der Luijt RB, Timmers HJLM, Hermus ARMM and Kempers MJE: Germline and somatic mosaicism in a family with multiple endocrine neoplasia type 1 (MEN1) syndrome. Eur J Endocrinol 180: K15-K19, 2019.

33. Matkar S, Thiel A and Hua X: Menin: A scaffold protein that controls gene expression and cell signaling. Trends Biochem Sci 38: 394-402, 2013.

34. Wang Y, Ozawa A, Zaman S, Prasad NB, Chandrasekharappa SC, Agarwal SK and Marx SJ: The tumor suppressor protein menin inhibits AKT activation by regulating its cellular localization. Cancer Res 71: 371-382, 2011.

35. Fang M, Xia F, Mahalingam M, Virbasius CM, Wajapeyee $N$ and Green MR: MEN1 is a melanoma tumor suppressor that preserves genomic integrity by stimulating transcription of genes that promote homologous recombination-directed DNA repair. Mol Cell Biol 33: 2635-2647, 2013

36. Lin SY and Elledge SJ: Multiple tumor suppressor pathways negatively regulate telomerase. Cell 113: 881-889, 2003.

37. Lawrence B, Blenkiron C, Parker K, Tsai P, Fitzgerald S, Shields P, Robb T, Yeong ML, Kramer N, James S, et al: Recurrent loss of heterozygosity correlates with clinical outcome in pancreatic neuroendocrine cancer. NPJ Genom Med 3: 18, 2018. 
38. Leoncini E, Carioli G, La Vecchia C, Boccia S and Rindi G: Risk factors for neuroendocrine neoplasms: A systematic review and meta-analysis. Ann Oncol 27: 68-81, 2016.

39. Ben Q, Zhong J, Fei J, Chen H, Yv L, Tan J and Yuan Y: Risk factors for sporadic pancreatic neuroendocrine tumors: A case-control study. Sci Rep 6: 36073, 2016.

40. Gallo M, Ruggeri RM, Muscogiuri G, Pizza G, Faggiano A and Colao A; NIKE Group: Diabetes and pancreatic neuroendocrine tumours: Which interplays, if any? Cancer Treat Rev 67: 1-9, 2018.

41. Fan Z, Gong Y, Huang Q, Yang C, Cheng H, Jin K, Fan K, Ni Q, Yu X, Luo G and Liu C: Diabetes is associated with the metastasis of pancreatic neuroendocrine tumors. Pancreas 49: 751-756, 2020.

42. Maxwell KN and Domchek SM: Cancer treatment according to BRCA1 and BRCA2 mutations. Nat Rev Clin Oncol 9: 520-528, 2012.

43. Sharma MB, Carus A, Sunde L, Hamilton-Dutoit S and Ladekarl M: BRCA-associated pancreatico-biliary neoplasms: Four cases illustrating the emerging clinical impact of genotyping. Acta Oncol 55: 377-381, 2016.
44. Lin AL and Gutmann DH: Advances in the treatment of neurofibromatosis-associated tumours. Nat Rev Clin Oncol 10: 616-624, 2013.

45. Gutmann DH, Ferner RE, Listernick RH, Korf BR, Wolters PL and Johnson KJ: Neurofibromatosis type 1. Nat Rev Dis Primers 3: 17004, 2017.

46. Frost M, Lines KE and Thakker RV: Current and emerging therapies for PNETs in patients with or without MEN1. Nat Rev Endocrinol 14: 216-227, 2018

47. Perren A, Wiesli P, Schmid S, Montani M, Schmitt A, Schmid C, Moch $\mathrm{H}$ and Komminoth P: Pancreatic endocrine tumors are a rare manifestation of the neurofibromatosis type 1 phenotype: Molecular analysis of a malignant insulinoma in a NF-1 patient. Am J Surg Pathol 30: 1047-1051, 2006.

(c) (i) $\Theta$ This work is licensed under a Creative Commons EY No ND Attribution-NonCommercial-NoDerivatives 4.0 International (CC BY-NC-ND 4.0) License. 